\title{
Long-term immunogenicity of hepatitis B vaccine and impact of a booster dose on health care students
}

\author{
Behzad Bijani ${ }^{1}$, Abbas Allami*1 $^{*}$ Farzaneh Jafari $^{1}$, Fatemeh Hajmanoochehri ${ }^{2}$, Soroush Bijani ${ }^{3}$
}

Received: 29 Dec 2017

Published: 20 March 2019

Abstract

Background: Accidental exposure to sharp instruments is an important problem for health care students. Thus, the aim of this study was to determine the rate of immunity in health care students 2 decades after national neonatal hepatitis B (HB) vaccination.

Methods: All junior students attending medicine, nursing and midwifery schools were screened for anti-HBs. One dose of hepatitis B vaccine was offered to all participants who did not have antibodies to HB surface antigen (anti-HBs) of $>10$ IU/L; then, they were tested for anti-HBs after a month. The participants were classified into 3 groups: postboosting nonimmune, postboosting immune, and preboosting immune. Chi square test and ANOVA were used for data analysis.

Results: In the first step, $65.20 \%$ of participants did not show immunity, but after receiving a booster dose, only $6.0 \%$ remained nonimmune. The mean age of nonimmune students was significantly higher than that of students who had postboosting immune and preboosting immune status ( $\mathrm{p}=0.001$ and 0.002 , respectively). Also, the mean injection time from last shot was higher in postboosting immune group compared to preboosting immune group $(\mathrm{p}<0.001)$. Also, prebooster anti-HBs level was significantly different among participants with suboptimal response and those who developed anamnestic response, indicating preserved immune memory $(\mathrm{p}=0.001)$.

Conclusion: High anamnestic response to HBV booster dose indicates sufficient immunity to HBV in the majority of health care students. However, identifying students who cannot respond to a booster dose of vaccine seems to be necessary at the beginning of health care courses.

Keywords: Hepatitis B, Vaccination, Medical student, Immunologic memory

\section{Conflicts of Interest: None declared}

Funding: Deputy for Research of Qazvin University of Medical Sciences

*This work has been published under CC BY-NC-SA 1.0 license.

Copyright $\odot$ Iran University of Medical Sciences

Cite this article as: Bijani B, Allami A, Jafari F, Hajmanoochehri F, Bijani S. Long-term immunogenicity of hepatitis B vaccine and impact of a booster dose on health care students. Med J Islam Repub Iran. 2019 (20 March);33:20. https://doi.org/10.47176/mjiri.33.20

\section{Introduction}

It is estimated that 360 million people are living with hepatitis B virus (HBV) worldwide (1). HBV causes nearly 700000 deaths each year globally (2). The World Health Organization (WHO) recommends that all infants and newborns even in countries with an intermediate or low prevalence of hepatitis should be

Corresponding author: Dr Abbas Allami, allami@qums.ac.ir

1. Department of Infectious Diseases, Qazvin University of Medical Sciences, Qazvin Iran

2. Department of Pathology, Qazvin University of Medical Sciences, Qazvin, Iran

3. Department of Pharmaceutical Biotechnology, Zanjan University of Medical Sciences, Zanjan, Iran immunized (3). Iran is a country with a moderate prevalence of hepatitis $B$. National neonatal vaccination was started in 1993 in Iran (4). Newborns who were vaccinated in the 1990s are now young adults entering medical, nursing, and midwifery schools. The health care personnel (HCP) are at particularly high risk of

$\uparrow$ What is "already known" in this topic:

Accidental exposure to sharp instruments is an important problem for health care students in teaching hospitals. In countries with national neonatal vaccination programs, the majority of health care students had lower antibody titer than the protective level at the beginning of health care courses.

$\rightarrow$ What this article adds:

The high anamnestic response to a booster dose indicates sufficient immunity to hepatitis B in the majority of health care students, but as the risk of exposure may be high in the forthcoming professional life, identifying students who cannot respond to a booster dose of vaccine seems to be necessary at the beginning of health care courses. 
infection with hepatitis B virus, primarily due to their increased risk of exposure to sharp contaminated tools $(5,6)$. The incidence of needle stick injuries in HCP ranges from 1.4 to 9.5 per $100 \mathrm{HCP}$ annually, which results in $0.42 \mathrm{HBV}$ infections per 100 needle stick injuries $(7,8)$. It has been estimated that unsafe needle stick injuries are responsible for over 21 million HBV infections each year worldwide (9).

In Qazvin University of Medical Sciences, despite education and interventions, a recent study reported a rate of $53 \%$ for needle stick injury in nurses at least once in the past year (10). Handling accidental blood exposures of HCP is problematic. Postexposure prophylaxis for $\mathrm{HBV}$ requires the evaluation of several factors, such as hepatitis B surface antigen status of the source, awareness of the vaccination, and HBV immunity status of the exposed HCP. Some problems include initial assessment of HCP in emergency situations (especially if HBV immunity status of HCP is uncertain and the patient is HBsAg-positive or even uncertain), secondary follow-up, and administration of HB immunoglobin (HBIG) for the exposed person (for negative or unknown immune) $(11,12)$. Fear and anxiety about the possible consequences of an exposure and absence from work are other problems; therefore, all HCP should know their HBV immune status (13).

Long-term protection of vaccine is not recommended for the general population (14). However, all HCP must be tested for the presence of protective levels of antibodies, and the immunity status of junior students who were vaccinated during infancy or adolescence is important for avoiding occupational HBV transmission (15). The United States Centre for Disease Control and the Advisory Committee on Immunization Practices (ACIP) recommends hepatitis $\mathrm{B}$ immunization and confirmation of immunoprotection for all HCP (16). In persons vaccinated at birth, antibody levels of hepatitis B surface antigen (anti-HBs) in adulthood are known to be lower than those immunized late $(17,18)$. In addition, the exact duration of protection after childhood vaccination is not well understood (19). On the other hand, the necessity and cost-effectiveness of continuing screening for every new medical and nursing student born after adoption of neonatal immunization is not yet evident $(20,21)$.

The aim of this study was to determine the proportion of health care students vaccinated in childhood with anti-HBs levels $<10 \mathrm{IU} /$ to evaluate their immune response to a booster dose of hepatitis B vaccine. Also, this study aimed to compare the characteristics of students with or without a protective anti-HBs response after administration of this adulthood booster dose.

\section{Methods}

From March to May 2016, the levels of serum antiHBs were screened in all junior students (medicine, nursing, midwifery) of Qazvin University of Medical
Sciences, Iran. The participation rate for this study was $80 \%$. A written consent form was obtained from all participants. They were asked about questions about whether they had chronic HBV infected parents, their HBV vaccination status (time of receiving the initial dose, time of the last dose, more shots after primary vaccination), weight, height, and smoking status. Blood samples were collected from all participants. Serum was separated and stored at $0^{\circ} \mathrm{C}$. Next, anti-HBs were measured by anti-HBs enzyme-linked immunosorbent assay (ELISA) kit (Abott laboratories).

Participants were considered immune if they had anti-HBs of $>10 \mathrm{IU} / \mathrm{L}$, and an additional dose was offered to all participants who did not have anti-HBs of $>$ $10 \mathrm{IU} / \mathrm{L}$. If agreed, a single dose (20 microgram) of hepatitis B vaccine (Euvax B LG manufacturing, South Korea) was injected to them. Participants were tested for hepatitis B surface antibody (ie, anti-HBs) a month after receiving their booster vaccine. A positive immune response to the vaccine is defined as the development of hepatitis B surface antibody (anti-HBs) at a titer of $>10 \mathrm{IU} / \mathrm{L}$. The participants were classified into 3 groups of postboosting nonimmune, postboosting immune, and preboosting immune based on their primary anti-HBs and response to booster vaccine.

Continuous variables were expressed as mean \pm standard deviation (SD) and categorical variables as frequencies and percentages. Chi square or Fisher's exact tests were used for nominal categorical variables and for comparison of the differences among the 3 groups. Analysis of variance (ANOVA) followed by a post hoc analysis (Tukey HSD) was used in SPSS 21.0 statistical analysis software (IBM Inc., Chicago, IL). A $\mathrm{p}$ value less than 0.05 was considered statistically significant. This trial was registered at the Iranian Registry of Clinical Trials website (registration ID: IRCT201510142276N3). The study protocol was approved by the medical ethics committee of Qazvin University of Medical Sciences.

\section{Results}

A total of 204 participants, including 118 nursing, 42 medical, and 44 midwifery students, were enrolled. Neither of them had ever received blood products, had HBV-infected member in their family, nor had they evidence of immunosuppression. None of the participants were taking immunosuppressive medications at the time of study. All students had received at least one-shot dose of $\mathrm{HB}$ vaccine and about $90 \%$ were immunized at birth. Only $6(3.2 \%)$ and $2(1.1 \%)$ participants were smoker and diabetic, respectively. Sixteen participants were unwilling to continue the study, and thus data from 188 participants were analyzed. The mean age of the participants was $19.98 \pm 1.87$ years, and $130(69.1 \%)$ were male (Table 1$)$.

In the first step, 133 of 204 participants $(65.2 \%)$ were found to have low or undetectable anti-HBs titers (Fig. 1 ), but after booster doses of HB-Vax, only 7 out of 133 participants $(6.0 \%)$ remained nonimmune/suboptimal responders. The mean of anti-HBs 
Table 1. Distribution of protective immunity status among 188 study participants

\begin{tabular}{|c|c|c|c|c|c|c|}
\hline & & $\begin{array}{c}\text { Post-boosting } \\
\text { nonimmune }\end{array}$ & $\begin{array}{c}\text { Post-boosting } \\
\text { immune }\end{array}$ & $\begin{array}{l}\text { Preboosting } \\
\text { immune }\end{array}$ & Total & $\mathrm{p}$ \\
\hline & \multicolumn{6}{|c|}{ Number $(\%)$ or Mean \pm SD } \\
\hline Sex & $\begin{array}{l}\text { Male } \\
\text { Female }\end{array}$ & $\begin{array}{l}3(42.9) \\
4(57.1)\end{array}$ & $\begin{array}{l}77(70.0) \\
33(30.0)\end{array}$ & $\begin{array}{l}50(70.4) \\
21(29.6)\end{array}$ & $\begin{array}{c}130(69.1) \\
58(30.9)\end{array}$ & 0.307 \\
\hline Marital status & $\begin{array}{c}\text { Single } \\
\text { Married }\end{array}$ & $\begin{array}{c}7(100.0) \\
0(0.0)\end{array}$ & $\begin{array}{c}104(95.4) \\
5(4.6)\end{array}$ & $\begin{array}{c}62(87.3) \\
9(12.7)\end{array}$ & $\begin{array}{c}173(92.5) \\
14(7.5)\end{array}$ & 0.098 \\
\hline Discipline of study & $\begin{array}{l}\text { Nursing } \\
\text { Medicine } \\
\text { Obstetric }\end{array}$ & $\begin{array}{l}4(57.1) \\
1(14.3) \\
2(28.6)\end{array}$ & $\begin{array}{l}58(52.7) \\
25(22.7) \\
27(24.5)\end{array}$ & $\begin{array}{l}48(67.6) \\
12(16.9) \\
11(15.5)\end{array}$ & $\begin{array}{l}110(58.5) \\
38(20.2) \\
40(21.3)\end{array}$ & 0.363 \\
\hline $\begin{array}{l}\text { Smoker } \\
\text { Diabetes mellitus } \\
\text { History of low birth } \\
\text { weight }\end{array}$ & & $\begin{array}{l}1(14.3) \\
0(0.0) \\
0(0.0)\end{array}$ & $\begin{array}{l}3(2.7) \\
2(1.8) \\
7(6.4)\end{array}$ & $\begin{array}{l}2(2.8) \\
0(0.0) \\
3(4.2)\end{array}$ & $\begin{array}{c}6(3.2) \\
2(1.1) \\
10(5.3)\end{array}$ & $\begin{array}{l}0.235 \\
0.488 \\
0.670\end{array}$ \\
\hline $\begin{array}{l}\text { Age (year) } \\
\mathrm{BMI}\left(\mathrm{Kg} / \mathrm{M}^{2}\right) \\
\text { Injection time } \\
\text { from last shot (year) }\end{array}$ & & $\begin{array}{l}22.43 \pm 6.05 \\
21.53 \pm 3.56 \\
20.17 \pm 0.98\end{array}$ & $\begin{array}{l}19.88 \pm 0.97 \\
22.17 \pm 3.12 \\
19.84 \pm 0.93\end{array}$ & $\begin{array}{l}19.89 \pm 2.04 \\
22.30 \pm 2.63 \\
17.62 \pm 4.96\end{array}$ & $\begin{array}{l}19.98 \pm 1.88 \\
22.20 \pm 2.95 \\
19.01 \pm 3.31\end{array}$ & $\begin{array}{c}0.002 * \\
0.799 \\
<0.001 *\end{array}$ \\
\hline $\begin{array}{l}\text { Initial anti-HBs titer } \\
\text { (IU/L) }\end{array}$ & & $0.26 \pm 0.66$ & $2.80 \pm 2.87$ & - & - & $<0.001^{*}$ \\
\hline
\end{tabular}

BMI: Body Mass Index, ${ }^{*} \mathrm{p}<0.05$

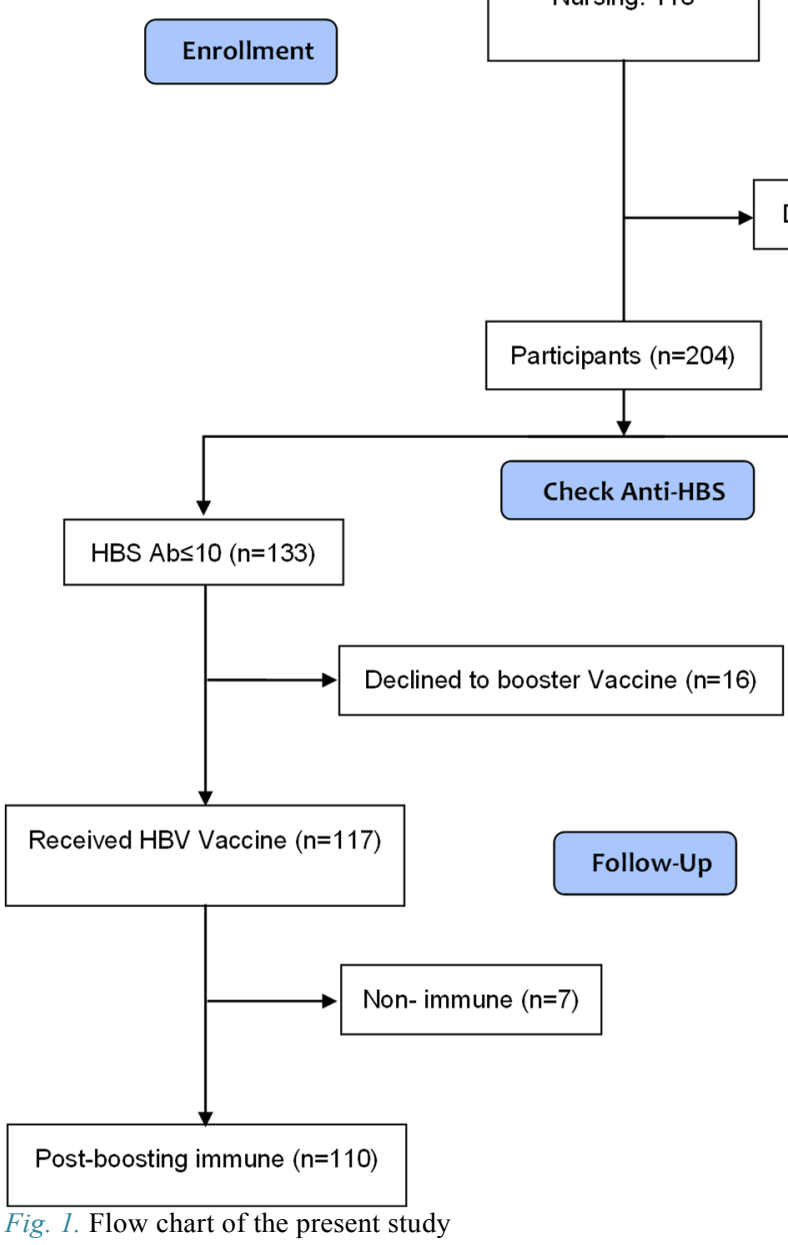

Declined to participate $(n=6)$

antibody in the 2 groups were $0.26 \pm 0.66 \mathrm{IU} / \mathrm{L}$ and $2.80 \pm 2.87 \mathrm{IU} / \mathrm{L}$, respectively, $\mathrm{p}<0.001$. The Tukey
HSD showed that the mean age of participants among nonimmune students $(22.43, \mathrm{SD}=6.05)$ was significant- 
ly higher than students who had postboosting immune (19.88, $\mathrm{SD}=0.97, \mathrm{p}=0.001)$ and preboosting immune status (19.89, $\mathrm{SD}=2.039, \mathrm{p}=0.002$ ) (Table 2).

Also, ANOVA for injection time from the last shot showed significant differences between the study groups $(p<0.001)$. The Tukey HSD showed that the mean of injection time from the last shot was higher at postboosting immune group compared to preboosting immune group $(19.84 \pm 0.93$ vs $17.62 \pm 4.95, \mathrm{p}<0.001)$ (Table 2). In the second step, Mann-Whitney test showed a significant difference between participants with suboptimal response (HBs-antibody $<10 \mathrm{IU} / \mathrm{L}$ ) and those who developed anamnestic response, indicating preserved immune memory $(\mathrm{p}=0.001)$.

The overall prevalence of true adverse reactions was low. Side-effects of the vaccine were limited to 6 cases with local symptoms [wheal ( 2 cases) and itching at the site of administration ( 4 cases)] and 3 cases with general symptoms [fever ( 2 cases) and myalgia ( 1 case)].

\section{Discussion}

In this study, approximately $65 \%$ of participants had low or undetectable anti-HBs titers; and after booster doses, only $3.0 \%$ of all participants remained nonimmune/suboptimal responders. Also, age (the time since last vaccination) was associated with a lower antibody level. These results are consistent with those of another study conducted in our institution in which half of the participants had low or undetectable anti-HBs titers (17).

Also, several studies showed that although the antibody response to hepatitis B in childhood vaccination is very high and reaches $98 \%$, the antibody level declines rapidly soon after, and protective level is detectable in a minority of vaccinees after 20 years $(22,23)$.

Most authorities believe that the diminished levels of anti-HBs do not necessarily indicate loss of immunity (24) and immunological memory in otherwise healthy adults vaccinated in infancy may be preserved for decades after vaccination. Thus, booster dose is not neces- sary even in those with low antibody levels (25). On the other hand, some believe that in HCP vaccinated in infancy who have a nonprotective anti-HBs level, the immune system may become overwhelmed by a highly infectious HBV inoculum during a long professional life (26).

Anti-HBs $\geq 10 \mathrm{IU} / \mathrm{L}$ is protective for people at risk of this infection $(27,28)$. In the present study, $34.8 \%$ of students had this level of antibody about 2 decades after vaccination in infancy. This result is consonant with the findings reported by previous studies that a minority of individuals vaccinated in childhood are fully protected against hepatitis $\mathrm{B}$ in adulthood with antibody level above $10 \mathrm{IU} / \mathrm{L}$. In a study in 420 adolescents aged 1619 years, born in the United States, who had received the primary hepatitis $B$ vaccine series, the protective level of antibody was detected in $24.1 \%$ of participants (29). In the mentioned study, the seroprotection rate was significantly higher in participants who received the first dose of vaccine before the seventh day after birth compared to participants who received the first dose 4 weeks after birth (33.9\% and $16.7 \%$, respectively). In studies performed on younger age groups, it was found that after childhood vaccination series, the obtained seroprotection rate was higher, as in a study in Egypt that found among participants aged 9 months to 16 years, $57.7 \%$ had antibody level $\geq 10 \mathrm{IU} / 1$. In that study, only $22.5 \%$ of the participants were over 15 years (30). Conversely, in another study in Naples, Italy, the rate of seroprotective antibody level was very high in university students compared to other studies, as $77 \%$ of the students vaccinated in infancy and $87.9 \%$ of students vaccinated at 12 years old had anti-HBs $\geq 10$ $\mathrm{IU} / \mathrm{L}$ (26). However, the reason for this discrepancy is not clear. These differences could be attributed to different vaccines or scheme of vaccination.

In the present study, $94.7 \%$ of participants with a low antibody response showed a protective level of antibody after receiving a booster dose of hepatitis $B$ vaccine. So, the overall protection reached $96.8 \%$ with a single booster dose. An increase in anti-HBs titer de-

Table 2. Multiple comparisons (Tukey HSD) of study groups

\begin{tabular}{|c|c|c|c|c|}
\hline \multirow[t]{2}{*}{ Dependent variable } & \multicolumn{4}{|c|}{ Immunity status (End of the study) } \\
\hline & (I) & $(\mathrm{J})$ & Mean difference (I-J) & $\mathrm{p}$ \\
\hline \multirow[t]{6}{*}{ Age } & Nonimmune & Postboosting immune & $2.547 *$ & $0.001 *$ \\
\hline & & Preboosting immune & $2.541^{*}$ & $0.002 *$ \\
\hline & Postboosting immune & Nonimmune & $-2.547^{*}$ & $0.001 *$ \\
\hline & & Preboosting immune & -0.006 & 1.00 \\
\hline & Preboosting immune & Nonimmune & $-2.541^{*}$ & $0.002 *$ \\
\hline & & Postboosting immune & 0.006 & 1.00 \\
\hline \multirow[t]{6}{*}{ BMI } & Nonimmune & Postboosting immune & -0.641 & 0.84 \\
\hline & & Preboosting immune & -0.770 & 0.79 \\
\hline & Postboosting immune & Nonimmune & 0.641 & 0.84 \\
\hline & & Preboosting immune & -0.129 & 0.96 \\
\hline & Preboosting immune & Nonimmune & 0.771 & 0.79 \\
\hline & & Postboosting immune & 0.129 & 0.96 \\
\hline \multirow{6}{*}{$\begin{array}{l}\text { Injection time } \\
\text { from last shot }\end{array}$} & Nonimmune & Postboosting immune & 0.326 & 0.97 \\
\hline & & Preboosting immune & 2.543 & 0.14 \\
\hline & Postboosting immune & Nonimmune & -0.326 & 0.967 \\
\hline & & Preboosting immune & $2.218^{*}$ & $<0.001^{*}$ \\
\hline & Preboosting immune & Nonimmune & -2.543 & 0.14 \\
\hline & & Postboosting immune & $-2.218^{*}$ & $<0.001^{*}$ \\
\hline
\end{tabular}


tected shortly after receiving a booster dose of the vaccine can be interpreted as an amnestic response due to preserved immune memory and guaranties adequate protection against future HBV infection $(14,18)$.

In a cohort study on Alaska Natives vaccinated 6 months after birth, 50\% had anti-HBs $>10$ IU/1 30 years later. Offering an intramuscular booster dose of hepatitis $B$ vaccine to individuals with anti-HBs $<10 \mathrm{IU} / \mathrm{L}$, $>90 \%$, shows evidence of seroprotection 3 decades after the primary vaccination series in the study population (20). Unfortunately, in most parts of the world, measuring the antibody response after childhood vaccination is not affordable. Thus, in the present study, there were no data on the primary protective response in the students, but the number of primary nonresponders must be very low. In another study in Bulgaria, a country with a moderate rate of hepatitis B virus infection, the majority of participants with a nonprotective antibody level showed an anamnestic antibody response by receiving a booster dose of vaccine. Thus, authors concluded that a booster dose is not recommended in national hepatitis B immunization programs (31).

In a study conducted in a territory hospital with high prevalence of hepatitis B in Southeast Asia, anamnestic response to a booster dose was detected in $95.8 \%$ of participants 2 decades after a 4 -dose childhood vaccination (32). Therefore, the evidence confirms that boosting with recombinant vaccine is not currently recommended for the general population in communities with a high prevalence of hepatitis B infection (33). At the same time, studies conducted in low prevalence communities also indicate that adulthood booster dose in the general population is not necessary (29).

In all the mentioned studies, the participants were not exclusively HCP. In a university in Hong Kong, the overall rate for anti-HBs $\geq 10 \mathrm{IU} / \mathrm{L}$ was $30.4 \%$ in medical and nursing students entering to health care disciplines who were vaccinated in infancy. In participants with HBs-antibody level $<10$ IU/L who received 3 booster doses of vaccine, anti-HBs antibody was measured at 30 days after the first and third booster doses. In this group, 30 days after the first booster dose, $85.5 \%$ of the participants had anti-HBs level $>10$ IU/L and the protective antibody level was achieved in all participants after the third dose. The authors recommended at least 3 booster doses of hepatitis B vaccine for all HCP vaccinated against hepatitis $B$ in childhood (21). In a study in Maryland, USA, from 159 HCP vaccinated in adulthood, $123(77 \%)$ had a protective antibody at least 10 years after vaccination. In the remaining participants, the protective level of antibody was achieved in $94 \%$ of the participants 3 weeks after receiving a booster dose and the total protective antibody rate reached $97 \%$ in the study population (34). The authors concluded that booster vaccination is not necessary in the otherwise healthy HCP. Thus, there is still controversy about the need for booster vaccination and the value of measuring antibody level before and after the booster doses of vaccine for HCP vaccinated in infancy (35).
Surprisingly, there is no consensus about prevaccination serological testing recommendations for medical and nursing students in the European Union, as these tests are not mandatory in the majority of countries. Among 5 countries with such regulations, only HBsantibody is checked in France, but in the United Kingdom, HBs antigen, anti-HBc antibody and even HBV DNA are recommended for medical students and HCP (36). In the present study, $3.7 \%$ of medical, nursing, and midwifery students did not show a protective level of antibody to childhood vaccination despite receiving a booster dose of hepatitis $B$ vaccine. As the risk of exposure to hepatitis B virus may be high in the forthcoming professional life, it is of paramount importance to identify students who do not respond to a booster dose of vaccine at the beginning of the study courses in medical disciplines.

In some studies, the response to a booster dose was not associated with the prebooster anti-HBs concentration (28). However, in the present study, the antibody response to the booster dose was significantly higher in participants with a higher prebooster antibody. Similarly, in the study of Salama et al, $98.1 \%$ of children with prebooster anti-HBs level $>3.3$ IU/L developed anamnestic response compared to $88.2 \%$ of those with lower antibody level $(\mathrm{p}<0.0001)(30)$.

The overall adverse effects of the vaccination for hepatitis B are low and the majority of them are local symptoms. Local pain and discomfort at the injection site, fatigue, irritability, and headache are seen in approximately one tenth of participants and $1 \%$ to $10 \%$ report fever, malaise, loss of appetite, nausea, vomiting, abdominal pain, and diarrhea $(37,38)$. However, more serious complications, including thrombocytopenia, neuritis, encephalitis, aseptic meningitis, erythema multiform, vasculitis, and anaphylactic reaction, are rare (39). The participants of this study did not report any severe adverse effects of vaccination and the only reported systemic symptom were fever in 1 student and myalgia in 2 .

This study had some limitations. The first limitation of this study was the lack of data about HBs antigen and anti-HBc antibody of the participants because of limited financial facilities. The second limitation was that the study was performed in 1 center only. Thus, future studies should be conducted in a multicenter setting so that the findings would be generalizable for clinical practice.

\section{Conclusion}

Results of this study showed sufficient immunity to HBV in the majority of health care students. However, it is of high importance to identify students who do not respond to a booster dose of vaccine at the beginning of the study courses in health care disciplines. An alternative strategy may be implementation of compulsory testing HBsAb 1 to 3 months after the completion of childhood vaccination. 


\section{Acknowledgement}

This study was supported by Deputy for Research of Qazvin University of Medical Sciences.

\section{Conflict of Interests}

The authors declare that they have no competing interests.

\section{References}

1. McMahon BJ. Chronic hepatitis B virus infection. Med Clin. 2014;98(1):39-54

2. Nayagam S, Thursz M, Sicuri E, Conteh L, Wiktor S, Low-Beer D, et al. Requirements for global elimination of hepatitis B: a modelling study. Lancet Infect Dis. 2016;16(12):1399-408.

3. Publication W. Hepatitis B vaccines: WHO position paperrecommendations. Vaccine. 2010;28(3):589-90.

4. Rezaee R, Aghcheli B, Poortahmasebi V, Qorbani M, Alavian SM, Jazayeri SM. Prevalence of national responsiveness to HBV vaccine after 22 years of Iranian Expanded Program on Immunization (EPI): A systematic review and meta-analysis study. Hepat Mon. 2015;15(5).

5. Hahné SJ, Veldhuijzen IK, Wiessing L, Lim T-A, Salminen M, van de Laar M. Infection with hepatitis $\mathrm{B}$ and $\mathrm{C}$ virus in Europe: a systematic review of prevalence and cost-effectiveness of screening. BMC Infect Dis. 2013;13(1):181

6. Walsh N, Verster A, Rodolph M, Akl EA. WHO guidance on the prevention of viral hepatitis $\mathrm{B}$ and $\mathrm{C}$ among people who inject drugs. Int J Drug Policy. 2014;25(3):363-71.

7. Askarian M, Yadollahi M, Kuochak F, Danaei M, Vakili V, Momeni M. Precautions for health care workers to avoid hepatitis B and C virus infection. Int J Occup Environ Med. 2011;2(4 October).

8. Elseviers MM, Arias-Guillén M, Gorke A, Arens HJ. Sharps injuries amongst healthcare workers: review of incidence, transmissions and costs. J Ren Care. 2014;40(3):150-6.

9. FitzSimons D, Francois G, De Carli G, Shouval D, Prüss-Üstün A, Puro V, et al. Hepatitis B virus, hepatitis C virus and other bloodborne infections in healthcare workers: guidelines for prevention and management in industrialised countries. J Occup Environ Med. 2008;65(7):446-51.

10. Mohammadi N, Allami A, Mohamadi RM. Percutaneous exposure incidents in nurses: Knowledge, practice and exposure to hepatitis B infection: Percutaneous exposure incidents in nurses. Hepat Mon. 2011;11(3):186.

11. Jagger J, Perry J, Gomaa A, Phillips EK. The impact of US policies to protect healthcare workers from bloodborne pathogens: the critical role of safety-engineered devices. J Infect Public Health 2008;1(2):62-71.

12. Zaidi MA, Beshyah SA, Griffiths RF. Needle stick injuries: An overview of the size of the problem, prevention and management. Ibnosina J Med Biomed Sci. 2009;2(2):53-61.

13. Worthington MG, Ross JJ, Bergeron EK. Posttraumatic stress disorder after occupational HIV exposure: two cases and a literature review. Infect Control Hosp Epidemiol. 2006;27(2):215-7.

14. Samandari T, Fiore AE, Negus S, Williams JL, Kuhnert W, McMahon BJ, et al. Differences in response to a hepatitis B vaccine booster dose among Alaskan children and adolescents vaccinated during infancy. Pediatrics. 2007;120(2):e373-e81.

15. Maltezou HC, Wicker S, Borg M, Heininger U, Puro V, Theodoridou M, et al. Vaccination policies for health-care workers in acute health-care facilities in Europe. Vaccine. 2011;29(51):9557-62.

16. Schillie S, Murphy TV, Sawyer M, Ly K, Hughes E, Jiles R, et al. $\mathrm{CDC}$ guidance for evaluating health-care personnel for hepatitis $\mathrm{B}$ virus protection and for administering postexposure management MMWR Recomm Rep. 2013;62(10):1-19.

17. Allami A, Mohammadi N, Najar A. Immunization Status Against Hepatitis B Among Iranian Junior Medical, Nursing, and Obstetrics Students With Different Vaccination Patterns. Biotech Health Sci. $2015 ; 2(3)$

18. Hammitt LL, Hennessy TW, Fiore AE, Zanis C, Hummel KB, Dunaway E, et al. Hepatitis B immunity in children vaccinated with recombinant hepatitis $\mathrm{B}$ vaccine beginning at birth: a follow-up study at 15 years. Vaccine. 2007;25(39):6958-64.

19. Keck JW, Bulkow LR, Raczniak GA, Negus SE, Zanis CL, Bruce
MG, et al. Hepatitis B virus antibody levels 7 to 9 years after booster vaccination in Alaska native persons. Clin Vaccine Immunol. 2014;21(9):1339-42.

20. Bruce MG, Bruden D, Hurlburt D, Zanis C, Thompson G, Rea L, et al. Antibody levels and protection after hepatitis $B$ vaccine: results of a 30-year follow-up study and response to a booster dose. J Infect Dis. 2016;214(1):16-22.

21. Chan PK, Ngai KL, Lao TT, Wong MC, Cheung T, Yeung AC, et al. Response to booster doses of hepatitis B vaccine among young adults who had received neonatal vaccination. 2014;9(9):e107163.

22. Schillie SF, Murphy TV. Seroprotection after recombinant hepatitis $B$ vaccination among newborn infants: a review. Vaccine. 2013;31(21):2506-16.

23. Yazdanpanah B, Safari M, Yazdanpanah S. Persistence of HBV vaccine's protection and response to hepatitis $B$ booster immunization in 5-to 7-year-old children in the Kohgiloyeh and Boyerahmad Province, Iran. Hepat Mon. 2010;10(1):17.

24. Dentinger CM, McMahon BJ, Butler JC, Dunaway CE, Zanis CL, Bulkow LR, et al. Persistence of antibody to hepatitis B and protection from disease among Alaska natives immunized at birth Pediatr Infect Dis J. 2005;24(9):786-92.

25. Leuridan E, Van Damme P. Hepatitis B and the need for a booster dose. Clin Infect Dis. 2011;53(1):68-75.

26. Coppola N, Corvino AR, De Pascalis S, Signoriello G, Di Fiore E, Nienhaus $A$, et al. The long-term immunogenicity of recombinant hepatitis B virus (HBV) vaccine: contribution of universal HBV vaccination in Italy. BMC Infect Dis. 2015;15(1):149.

27. Tohme RA, Awosika-Olumo D, Nielsen C, Khuwaja S, Scott J, Xing $\mathrm{J}$, et al. Evaluation of hepatitis B vaccine immunogenicity among older adults during an outbreak response in assisted living facilities. Vaccine. 2011;29(50):9316-20.

28. Zanetti A, Parlato A, Romanò L, Desole MG, Ferrera G, Giurdanella $F$, et al. Challenge with a hepatitis $B$ vaccine in two cohorts of 4-7-year-old children primed with hexavalent vaccines: An open-label, randomised trial in Italy. Vaccine. 2012;30(39):5770

29. Middleman AB, Baker CJ, Kozinetz CA, Kamili S, Nguyen C, Hu DJ, et al. Duration of protection after infant hepatitis $B$ vaccination series. Pediatrics. 2014;133(6):e1500-e7.

30. Salama I, Samim S, Salama S, Foud W, Abdel Hamid A, Said Z. Persistence of protection to hepatitis $B$ vaccine and response to booster dose among children and adolescents in Dakahleya-Egypt Egypt J Immunol. 2014;21(1):13-26.

31. Teoharov P, Kevorkyan A, Petrova N, Baltadzhiev I, Van Damme $\mathrm{P}$. Immune memory and immune response in children from Bulgaria 5-15 years after primary hepatitis B vaccination. Pediatr Infect Dis J. 2013;32(1):51-3.

32. Poovorawan Y, Chongsrisawat V, Theamboonlers A, Bock HL, Leyssen M, Jacquet J-M. Persistence of antibodies and immune memory to hepatitis $\mathrm{B}$ vaccine 20 years after infant vaccination in Thailand. Vaccine. 2010;28(3):730-6.

33. Zhu CL, Liu P, Chen T, Ni Z, Lu LL, Huang F, et al. Presence of immune memory and immunity to hepatitis $\mathrm{B}$ virus in adults after neonatal hepatitis B vaccination. Vaccine. 2011;29(44):7835-41.

34. Gara N, Abdalla A, Rivera E, Zhao X, Werner JM, Liang TJ, et al. Durability of antibody response against hepatitis B virus in healthcare workers vaccinated as adults. Clin Infect Dis. 2014;60(4):505-13.

35. De Schryver A, Claesen B, Meheus A, Hambach R, Van Sprundel M, François G. Hepatitis B vaccination policies for student healthcare workers in Europe. J Hosp Infect. 2014;86(2):147-50.

36. Halperin SA, McNeil S, Langley JM, Smith B, MacKinnonCameron D, McCall-Sani R, et al. Safety and immunogenicity of different two-dose regimens of an investigational hepatitis $\mathrm{B}$ vaccine (hepatitis B surface antigen co-administered with an immunostimulatory phosphorothioate oligodeoxyribonucleotide) in healthy young adults. Vaccine. 2012;30(36):5445-8.

37. Chaiklang K, Wipasa J, Chaiwarith R, Praparattanapan J, Supparatpinyo K. Comparison of immunogenicity and safety of four doses and four double doses vs. standard doses of hepatitis B vaccination in HIV-infected adults: a randomized, controlled trial. PLoS One. 2013;8(11):e80409.

38. Xie J, Zhao L, Zhou S, He Y. Statistical and Ontological Analysis of Adverse Events Associated with Monovalent and Combination Vaccines against Hepatitis A and B Diseases. Sci Rep. 2016;6. 